\title{
Pathological prognostic factors for post-resection survival in patients with hepatocellular carcinoma associated with non-alcoholic fatty liver disease
}

\author{
Mizelle D’Silva ${ }^{1}$, Hee Young $\mathrm{Na}^{2}$, Jai Young Cho ${ }^{1}$, Ho-Seong Han ${ }^{1}$, Yoo-Seok Yoon ${ }^{1}$, Hae Won Lee ${ }^{1}$, \\ Jun Suh Lee ${ }^{1}$, Boram Lee ${ }^{1}$, Moonhwan Kim ${ }^{1}$ \\ ${ }^{1}$ Department of Surgery, Seoul National University Bundang Hospital, Seoul National University College of Medicine, Seongnam-si, Korea; \\ ${ }^{2}$ Department of Pathology, Seoul National University Bundang Hospital, Seoul National University College of Medicine, Seongnam-si, Korea \\ Contributions: (I) Conception and design: JY Cho, HS Han, HY Na; (II) Administrative support: YS Yoon, HW Lee; (III) Provision of study materials \\ or patients: JY Cho, HS Han, YS Yoon; (IV) Collection and assembly of data: M D'Silva, B Lee, M Kim; (V) Data analysis and interpretation: M \\ D'Silva, B Lee, JS Lee; (VI) Manuscript writing: All authors; (VII) Final approval of manuscript: All authors. \\ Correspondence to: Jai Young Cho, MD, PhD. Department of Surgery, Seoul National University Bundang Hospital, Gumi-ro 173, Bundang-gu, \\ Seongnam-si, Gyeonggi-do, 13620, Korea. Email: jychogs@gmail.com.
}

Background: The incidence of hepatocellular carcinoma (HCC) in patients with non-alcoholic fatty liver disease (NAFLD) is steadily increasing. However, little is known about the characteristics of these patients or the factors affecting their prognosis. Our aim was to evaluate the pathological prognostic factors associated with survival in NAFLD patients.

Methods: This was a retrospective cohort study of 575 patients who underwent resection of HCC between January 2004 and December 2018. HCC was associated purely with NAFLD or hepatitis B virus (HBV) based on the pathology and viral markers. The pathological markers of HCC were compared between patients with pure NAFLD and patients with pure HBV.

Results: The pathological factors were similar between the two groups. There were no differences in overall survival $(\mathrm{OS} ; \mathrm{P}=0.283$ ) or recurrence-free survival $(\mathrm{RFS} ; \mathrm{P}=0.990)$ between the pure NAFLD and pure HBV groups. The NAFLD group had a similar local RFS $(\mathrm{P}=0.785)$ but a better systemic RFS compared with the $\mathrm{HBV}$ group, $(\mathrm{P}=0.089)$. In multivariable analysis using bootstrapping with resampling and replacement of data, no single factor was significantly associated with RFS. However, the Ki-67 labeling index $[\mathrm{P}=0.022$; bootstrap 95\% confidence interval (CI): 0.000-0.919] was the only independent factor associated with systemic recurrence in the NAFLD group.

Conclusions: In patients with HCC associated with NAFLD, the Ki-67 labeling index determined by immunohistochemistry may predict the risk of systemic recurrence following resection, allowing for stricter monitoring protocols for such patients.

Keywords: Ki-67; non-alcoholic fatty liver disease (NAFLD); hepatocellular carcinoma (HCC); p53; survival

Submitted Apr 23, 2021. Accepted for publication May 28, 2021.

doi: $10.21037 /$ tcr-21-707

View this article at: https://dx.doi.org/10.21037/tcr-21-707

^ ORCID: 0000-0003-4434-2128. 


\section{Introduction}

The advances provided by science and technology have also introduced some pitfalls and problems. In particular, there have been profound changes in the dietary habits of people worldwide, which have led to an epidemic of obesity and its co-morbidities, including metabolic syndrome and type 2 diabetes $(1,2)$. Non-alcoholic fatty liver disease (NAFLD) is becoming rampant, and its incidence is increasing in parallel with the increasing incidences of type 2 diabetes and metabolic syndrome $(3,4)$. No effective pharmacological therapy has been found for the treatment of steatohepatits (5). NAFLD has the potential to progress to hepatocellular carcinoma (HCC). Although HCC is commonly associated with hepatitis B virus (HBV) and hepatitis C virus (HCV), in recent years there has been a steady increase in the number of patients diagnosed with HCC associated with NAFLD. Currently, little is known about the characteristics of these patients or the factors that affect their prognosis. Therefore, it has become imperative to identify the factors associated with poor prognosis of HCC in patients with NAFLD. The criteria used to diagnose NAFLD are mainly based on pathological and clinical features (6). HCC is characterized by distinct pathological findings and immunohistochemical features. Some of the factors previously identified as prognosticators for HCC are serum AFP level, des-gamma-carboxy prothrombin level, tumor size and number, margin status, major vessel invasion, tumor stage, Edmonson-Steiner (ES) grade, Child-Pugh score, portal hypertension and cirrhosis. Immunohistochemical markers studied are keratin 19 (K19), epithelial cell adhesion molecule (EpCAM), and CD133 (7). No previous studies have shown the influence of the etiology of HCC on the risk for recurrence after resection. Therefore, we sought to determine the prognostic pathological factors for post-resection survival in patients with HCC associated with NAFLD.

We present the following article in accordance with the STROBE reporting checklist (available at https://dx.doi. org/10.21037/tcr-21-707).

\section{Methods}

The study was conducted in accordance with the Declaration of Helsinki (as revised in 2013). This retrospective analysis of patients who underwent surgical resection for HCC was conducted after approval from the institutional review board at Seoul National University Bundang Hospital (South Korea) (IRB No. B-2007/625/107). Individual consent for this study was waived. After pathological review, patients diagnosed with pure NAFLD or pure HBV were eligible for this study. NAFLD was diagnosed based on the scores of steatosis, lobular inflammation, and hepatocyte ballooning. Steatosis was scored as $0,1,2$, and 3 based on the observed macrovesicular steatosis $(<5 \%=0, \geq 5 \%$ to $<33 \%=1, \geq 33 \%$ to $<66 \%=2$, and $\geq 66 \%=3)$. Lobular inflammation was scored as $0,1,2$, or 3 if $0,<2,2-4$, or $\geq 4$ foci, respectively, were detected per field (magnification, 200×). Hepatocyte ballooning was graded as 0,1 , or 2 if there were none, a few, or many ballooned hepatocytes, respectively. The NAFLD activity score was calculated as the sum of these three scores (total score, 0-8 points) for each patient (6). A score of $\geq 3$ was classified as non-alcoholic steatohepatitis (8). Pure NAFLD was defined as alcohol intake $<20 \mathrm{~g} /$ day and no evidence of viral hepatitis (9). Pure HBV was defined as pathologic confirmation of chronic hepatitis together with viral markers for $\mathrm{HBV}$, in the absence of alcoholic intake (i.e., <20 g/day), other viral causes of hepatitis, and NAFLD.

The demographic characteristic (age, body mass index, and sex), preoperative factors [American Society of Anesthesiologists (ASA) score, co-morbidities, preoperative tumor size, and number of tumors], intraoperative findings (operation time and blood loss), postoperative factors (complications and hospital stay), and pathological factors [cirrhosis, margin status, microvascular invasion, serosal invasion, bile duct invasion, T staging, ES grade, and immunohistochemistry findings for cyclooxygenase-2 (COX2), Ki-67, and p53] were retrieved from medical records.

\section{Pathological evaluation}

All specimen slides were evaluated by two pathologists. The background liver was evaluated to confirm the presence of NAFLD or HBV. The presence and type of cirrhosis were evaluated microscopically. The tumor was then evaluated using gross and microscopic sections. Characteristics, including microvascular invasion, serosal invasion, and bile duct invasion were evaluated. The ES grade was noted and the margin status was evaluated. Immunochemistry was performed using 4- $\mu$ m-thick paraffin-embedded sections. Sections were stained with specific antibodies for $\mathrm{p} 53$ (DO-7, 1:1,000, Dako), Ki-67 (MIB-1, 1:200, Dako), and COX2 (COX229, 1:300, Invitrogen) using an automated immunostainer (BenchMark XT, Ventana Medical Systems, Tucson, AZ, USA) in accordance with the manufacturer's 
protocol. For Ki-67, cells with any intensity of nuclear staining were considered positive whereas only strong nuclear staining was considered positive for p53 (10). For COX2, cytoplasmic staining was considered positive. After counting 500-1,000 cells per slide, the percentages of hepatocytes positive for each antibody were calculated.

\section{Statistical analysis}

Data were tabulated on an Excel (Microsoft, Redmond, WA, USA) spreadsheet and statistical analyses were performed using SPSS for Windows version 20 (IBM, Chicago, IL, USA). Categorical data are presented as frequencies and percentages, and continuous data are presented as the mean \pm standard deviation. Between-group comparisons were conducted using logistic regression analysis. Survival curves were plotted using the Kaplan-Meier method and compared using the log-rank test. All tests were two-tailed and a $\mathrm{P}$ value of $<0.05$ was considered significant. For the survival analysis of patients in the NAFLD group, a $\mathrm{P}$ value of $<0.1$ was considered significant, this was due to the fact that very few factors showed a significance level of $<0.05$ so could not be used for multivariate analysis. Overall survival (OS) was calculated as the time from the date of surgery to the date of the last follow-up or death. Recurrence-free survival (RFS) was calculated as the time from the date of surgery to the date of recurrence or date of last follow-up whichever is earlier. Local RFS was calculated as the time from the date of surgery to the date of hepatic recurrence. Systemic RFS was calculated as the time from the date of surgery to the date of the first instance of extrahepatic progression. Prognostic factors in patients with NAFLD were validated using Cox regression analysis and a bootstrap procedure (2,000 times). Bootstrap validation is a method of random resampling with replacement from an original dataset for assessing statistical inference (11).

\section{Results}

Of 857 patients who underwent liver resection for HCC in the study period, 575 were eligible for this study. The pure NAFLD group comprised $36(6.3 \%)$ patients and the pure HBV group comprised 539 (93.7\%) patients.

\section{Pathological characteristics}

The pathological characteristics of both groups are displayed in Table 1. Cirrhosis was present in both groups of patients, and the majority of patients in the NAFLD group had a mixed type of cirrhosis $(\mathrm{n}=17,89.5 \%)$ and none had pure micronodular cirrhosis $(\mathrm{P}=0.039)$. Gross pathology revealed an expanding nodular type in half of the patients in each group, and none of the patients in the NAFLD group showed evidence of the gross infiltrative or trabecular types. Both groups were similar in terms of the proportions of patients with benign findings $(\mathrm{P}=0.998)$ and a positive resection margin $(\mathrm{P}=0.462)$. The majority of patients in both groups had a solitary tumor $(\mathrm{P}=0.679)$ with similar numbers of patients with small tumors $(<2 \mathrm{~cm})$. Furthermore, there were no differences in the proportions of patients with satellite nodules $(\mathrm{P}=0.786)$, microvascular invasion $(\mathrm{P}=0.359)$, and serosal invasion $(\mathrm{P}=0.635)$. The ES tumor grade $(\mathrm{P}=0.806)$ and the pathological $\mathrm{T}$ stage $(\mathrm{P}=0.745)$ were also similar in both groups. In terms of immunohistochemistry findings, there were no differences in $553(\mathrm{P}=0.892)$, COX2 $(\mathrm{P}=0.784)$, and $\mathrm{Ki}-67(\mathrm{P}=0.548)$ expression levels between the two groups (Table 1).

\section{Factors affecting survival}

The patients were followed up for a median of 42 [21$75]$ months. There was no difference in the OS between the pure NAFLD and the pure HBV group $(\mathrm{P}=0.283)$. However, the systemic RFS was greater in the pure NAFLD group compared to the pure HBV group, $(\mathrm{P}=0.089$, Figure 1). There was no difference in the overall RFS $(\mathrm{P}=0.925)$ and local RFS $(\mathrm{P}=0.877)$ between both groups (Figure 2). The 5-year OS and RFS was $84.6 \%$ and $42 \%$ in the NAFLD group versus $75.3 \%$ and $41.8 \%$ in the HBV group respectively. In the univariate analyses of all patients with HCC, pathological factors associated with 5-year RFS were cirrhosis $(\mathrm{P}=0.008)$, positive resection margin $(\mathrm{P}=0.004)$, microvascular invasion $(\mathrm{P}<0.001)$, serosal invasion $(\mathrm{P}=0.002)$, ES grade $(\mathrm{P}=0.030)$, $\mathrm{T}$ stage $(\mathrm{P}<0.001)$, and Ki-67 $>15 \%(\mathrm{P}=0.001)$ (Table 2$)$. In the multivariable analysis, positive resection margin $[\mathrm{P}=0.006$; odds ratio (OR): $2.047,95 \%$ confidence interval (CI): $1.226-$ 3.416], T stage ( $\mathrm{P}=0.002$; OR: 1.815, 95\% CI: 1.255-2.626), microvascular invasion $(\mathrm{P}<0.001$; OR: $1.794,95 \% \mathrm{CI}$ : 1.375-2.340), and $\mathrm{Ki}-67>15 \%(\mathrm{P}=0.048$; OR: $1.310,95 \%$ CI: 1.002-1.713) were associated with 5-year RFS (Table 3).

The pathological factors associated with 5 -year OS were microvascular invasion $(\mathrm{P}<0.001)$, serosal invasion $(\mathrm{P}=0.003)$, ES grade $(\mathrm{P}=0.016)$, $\mathrm{T}$ stage $(\mathrm{P}<0.001)$, and Ki-67 >15\% ( $\mathrm{P}<0.001)$ (Table 2). The factors associated with 5 -year OS in the multivariable analysis were $\mathrm{T}$ stage ( $\mathrm{P}=0.015$; OR: 1.867, 95\% CI: 1.128-3.091), microvascular 
Table 1 Pathological characteristics of patients in the HBV and NAFLD groups

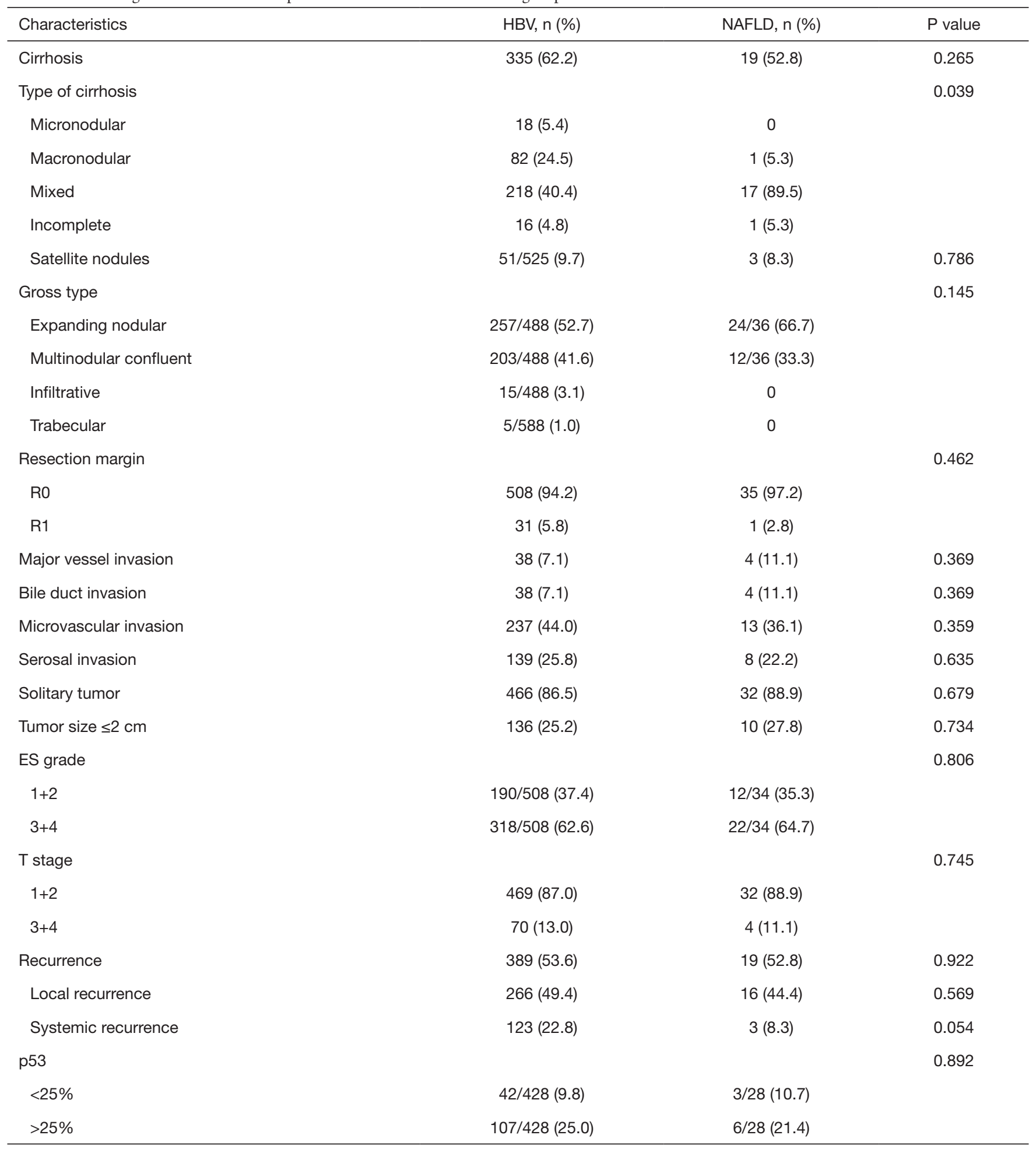

Table 1 (continued) 
Table 1 (continued)

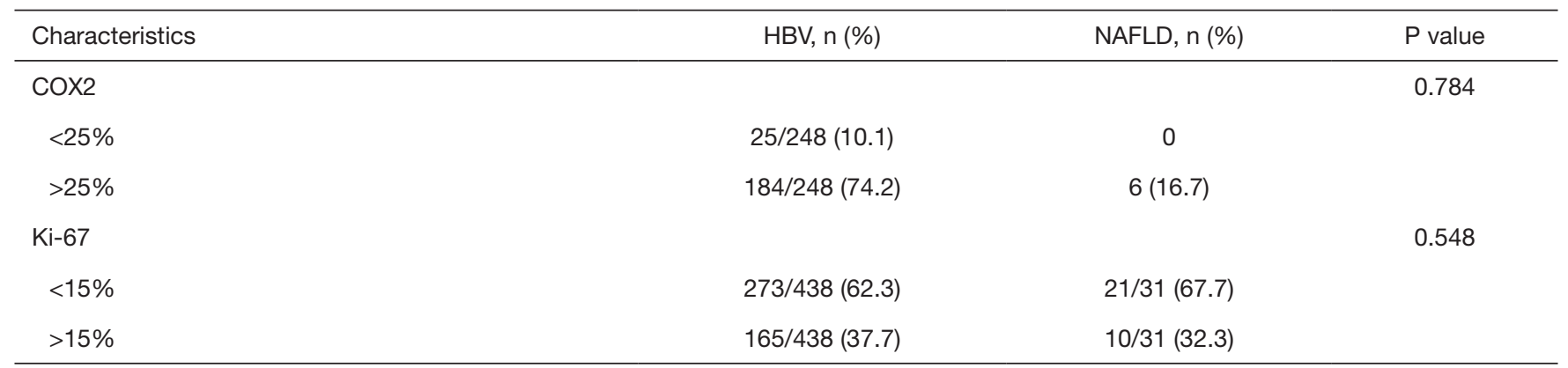

Values are $\mathrm{n}(\%)$. HBV, hepatitis B virus; NAFLD, non-alcoholic fatty liver disease; ES, Edmonson-Steiner; COX2, cyclooxygenase-2.
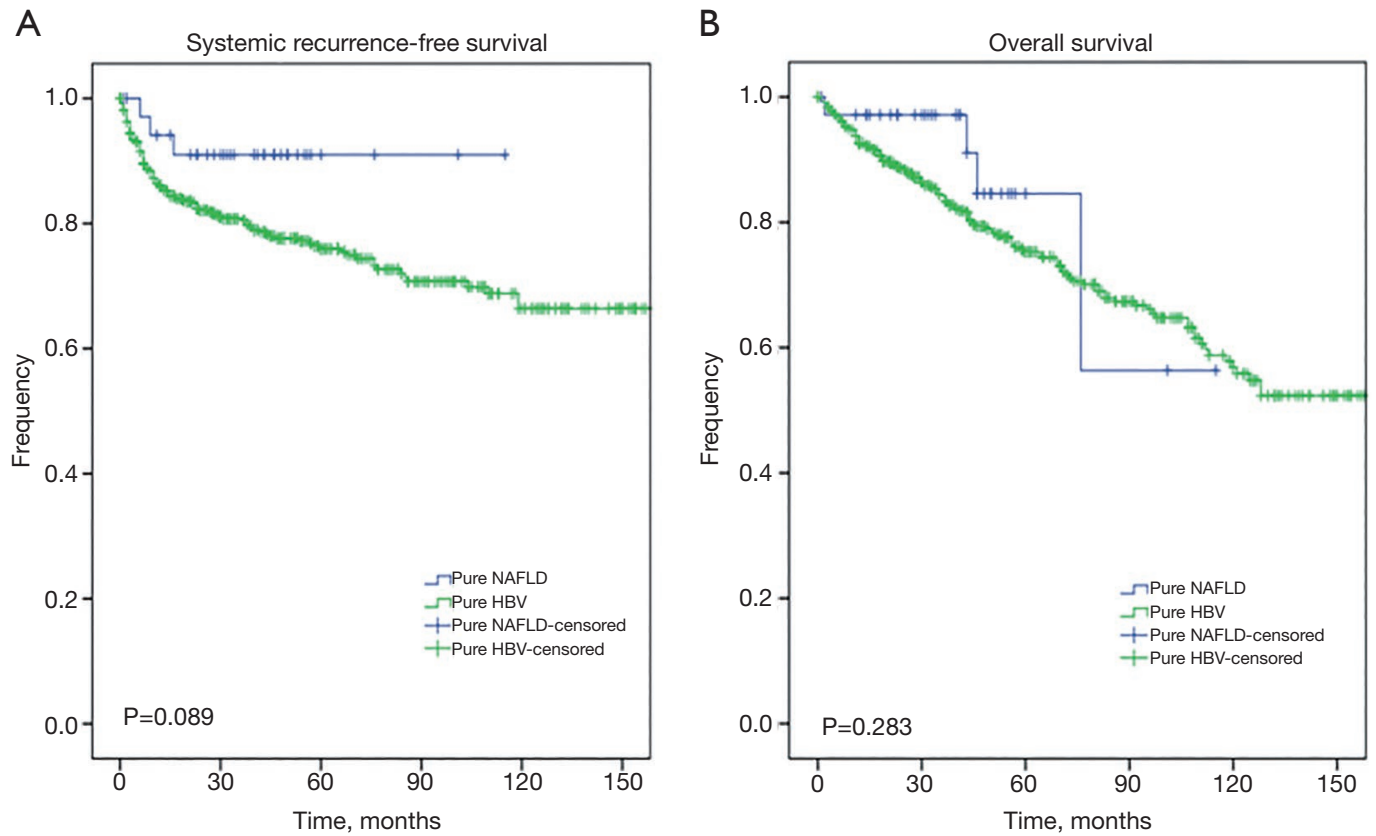

Figure 1 Systemic RFS (A) and OS (B) curves for patients with HCC associated purely with NAFLD or HBV. RFS, recurrence-free survival; OS, overall survival; HCC, hepatocellular carcinoma; NAFLD, non-alcoholic fatty liver disease; HBV, hepatitis B virus.

invasion ( $\mathrm{P}=0.003$; OR: $1.861,95 \% \mathrm{CI}: 1.227-2.823)$, and Ki-67 >15\% (P=0.023; OR: 1.586, 95\% CI: 1.065-2.361) (Table 3).

\section{Factors affecting survival in patients with NAFLD}

On analyzing NAFLD patients for pathological factors affecting survival, cirrhosis $(\mathrm{P}=0.084)$, ES grade $(\mathrm{P}=0.054)$, $\mathrm{T}$ stage $(\mathrm{P}=0.019), \mathrm{Ki}-67>15 \%(\mathrm{P}=0.071)$, and $\mathrm{p} 53$ $(\mathrm{P}=0.038)$ were associated with 5 -year RFS, but no pathological factors were associated with 5 -year OS. After performing multivariable analysis using bootstrapping with resampling and replacement of data up to 2,000 times, none of the variables were significantly associated with RFS.

Recurrence was then divided into local recurrence and systemic recurrence (Table 4). None of the variables were significantly associated with local RFS. However, for systemic RFS, the univariate analyses revealed that hypertension $(\mathrm{P}=0.067)$, serosal invasion $(\mathrm{P}=0.085)$, $\mathrm{T}$ stage $(\mathrm{P}<0.001)$ and $\mathrm{Ki}-67(\mathrm{P}=0.021)$ were significant variables (Figure 3). In the multivariable analysis with the bootstrapping method, Ki-67 ( $\mathrm{P}=0.022$; bootstrap 95\% CI: $0.000-0.919)$ was the only independent variable associated with systemic recurrence in patients with NAFLD. 
A

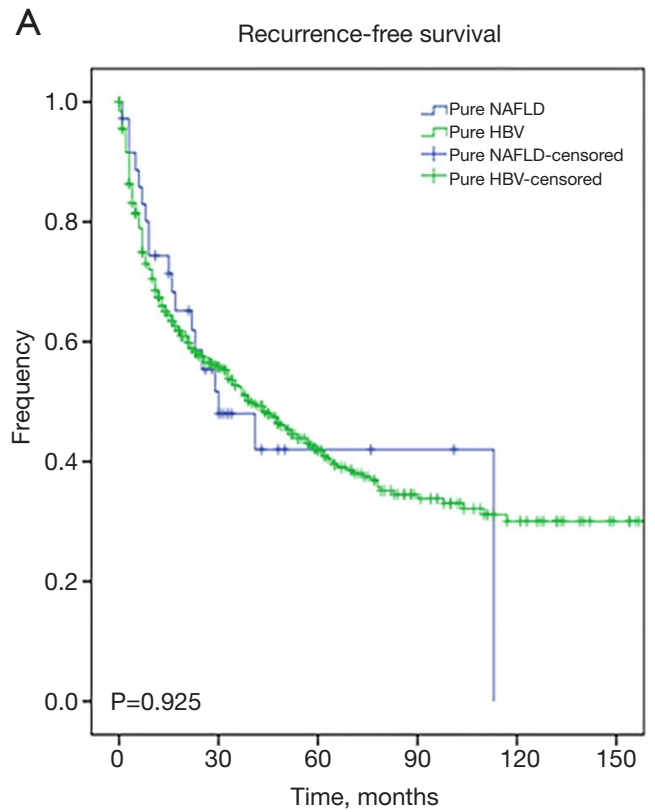

B

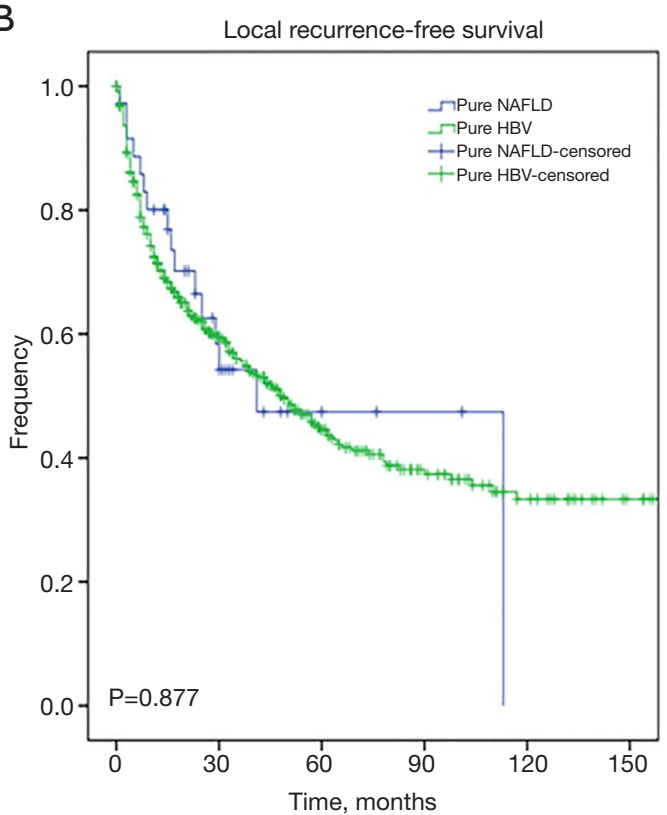

Figure 2 Overall RFS (A) and local RFS (B) curves for patients with HCC associated purely with NAFLD or HBV. RFS, recurrence-free survival; HCC, hepatocellular carcinoma; NAFLD, non-alcoholic fatty liver disease; HBV, hepatitis B virus.

\section{Discussion}

HCC is the second leading cause of cancer-related deaths worldwide (12). HCC commonly occurs in patients with viral hepatitis (13). However, with recent increase in the incidence of NAFLD, the number of patients with HCC associated- NAFLD has also increased. Various methods, including systemic agents, radiofrequency ablation, transarterial chemoembolization, microwave ablation, radiotherapy, surgical resection, and liver transplantation are available for the treatment of HCC $(14,15)$. Surgical resection is the preferred treatment considered curative for HCC (16). HCC has a distinct set of histological and immunological characteristics (7). Various studies have analyzed the impact of molecular markers on the prognosis of HCC (17-19), but there is no data regarding the prognostic relevance of these molecular markers in patients with HCC associated with NAFLD.

The frequency of cirrhosis was similar in the NAFLD and HBV groups in this study, and the development of cirrhosis is not critical for the development of HCC in patients with NAFLD (20). None of the patients in the NAFLD group had a purely micronodular pattern of cirrhosis; instead, the mixed pattern was the most common pattern found. Other pathological variables, including the ES grade, microvascular invasion, and serosal invasion, were similar in both groups. Furthermore, there were no differences in the immunohistochemistry markers between the groups. Of note, the 5-year RFS and OS rates were similar in both groups. While the 5-year local RFS rate was similar in both groups, the systemic RFS was better in the NAFLD group. Microvascular invasion and Ki-67 >15\% were identified as prognostic factors associated with RFS in patients with HCC. In addition to these factors $T$ stage was associated with OS. However, there were no independent prognostic factors in patients with NAFLD. When we analyzed local and systemic RFS, we found that Ki-67 was the only independent prognostic pathological factor associated with systemic RFS.

$\mathrm{Ki}-67$ is a protein named after the city in which it was first discovered (Kiel) and the number of the original clone. It was initially believed to be an antigen but was later identified as a protein (21). Positivity for Ki-67 is a measure of cellular growth because it is expressed during active growth phases and absent in the rest phase (22). The $\mathrm{Ki}-67$ labeling index, defined as the percentage of cells positive for Ki-67, provides a useful insight into the growth characteristics of the tumor as well as the potential outcome of patients because it represents the physiological status of cellular proliferation (23). A recent meta-analysis revealed that Ki-67 is strongly associated with the aggressiveness of 
Table 2 Univariate analyses of factors associated with survival in patients with HCC

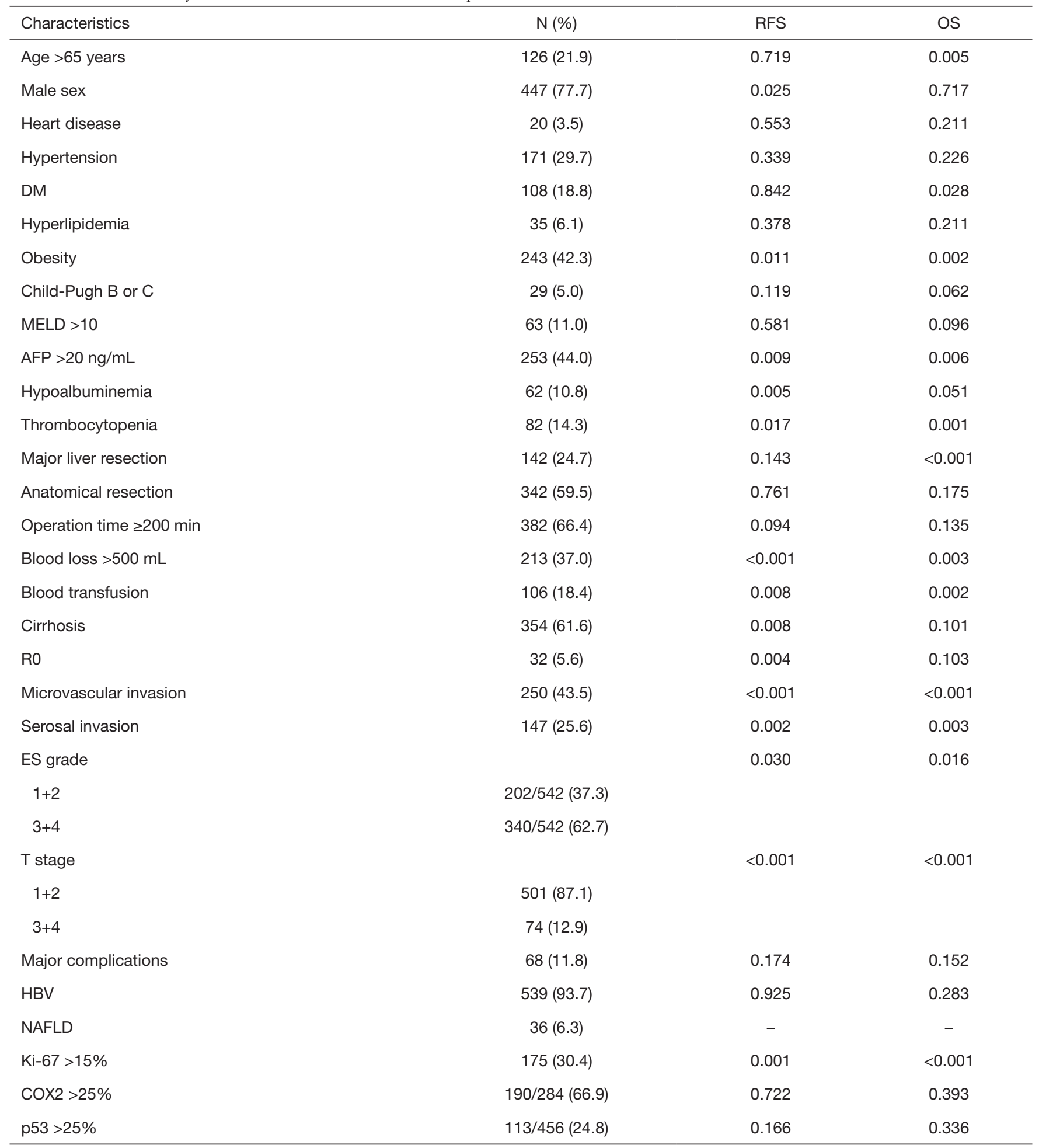

HCC, hepatocellular carcinoma; RFS, recurrence-free survival; OS, overall survival; DM, diabetes mellitus; MELD, Model for End-Stage Liver Disease; AFP, $\alpha$-fetoprotein; ES, Edmonson-Steiner; COX2, cyclooxygenase-2. 
Table 3 Multivariable analyses of factors associated with survival in patients with HCC

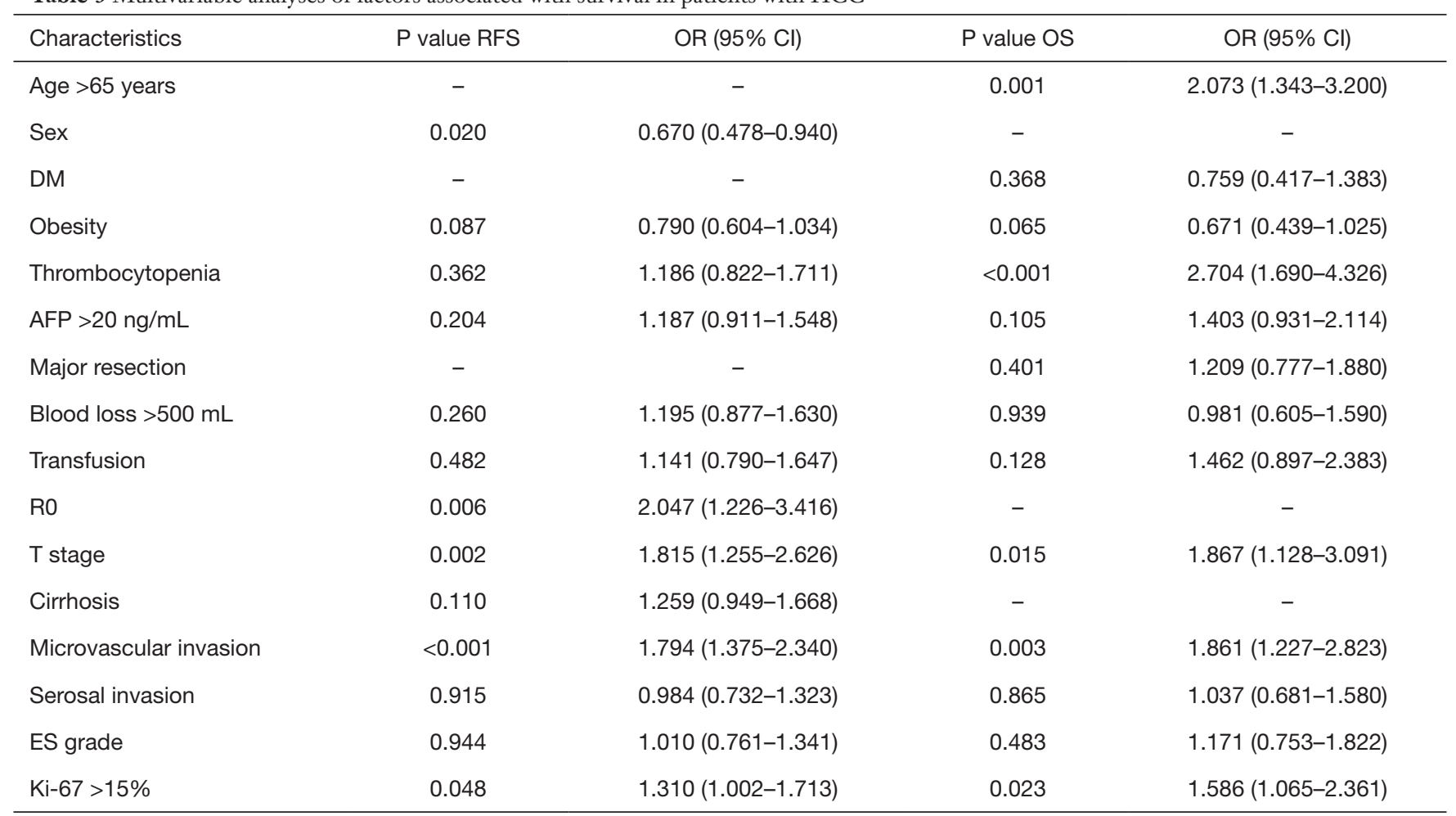

HCC, hepatocellular carcinoma; RFS, recurrence-free survival; OR, odds ratio; Cl, confidence interval; OS, overall survival; DM, diabetes mellitus; AFP, $\alpha$-fetoprotein; ES, Edmonson-Steiner.

the tumor. A high Ki-67 labeling index was associated with the potential deterioration of patients with HCC (24). In contrast to our findings, El-Bendary et al. reported that the Ki-67 proliferation index was greater in patients with $\mathrm{HBV}$ than in patients with NAFLD (22). However, Hussein et al. reported that the proliferation index was similar in patients with NAFLD and patients with HCV (25).

Several studies have shown that the Ki-67 labeling index is a prognostic factor for recurrence and OS in patients with HCC $(26,27)$. However, most of these earlier studies included a mixed population. The molecular markers for prognosis may differ between the different etiologies of HCC, including HBV, HCV, NAFLD, and alcoholic liver disease. Hence, studies involving mixed populations may provide inaccurate results (28). Hence we decided to study $\mathrm{Ki}-67$ as a predictor for patients with NAFLD associated HCC and did not analyze patients in the HBV group in this respect. Although $\mathrm{Ki}-67$ appeared to have prognostic roles for OS and RFS in our analyses of all patients with HCC, this was not apparent in the analyses of patients with pure NAFLD. The systemic recurrence rate was much lower in the NAFLD group than in the HBV group. When we studied NAFLD patients in detail, what was interesting to note was that even though $\mathrm{Ki}-67$ was not significantly related to OS and RFS, it was found to be a prognostic marker for systemic recurrence.

The serum p53 levels is another marker associated with poor prognosis in patients with HCC (29) and studies have demonstrated the association of $\mathrm{p} 53$ with RFS (30). HCCs with mutated p53 show high malignant potential, and p53 mutations in the primary lesion can indicate the biological behavior of recurrent HCCs. It is also a useful independent prognostic factor associated with survival after recurrence (31). In a recent prospective study, p53 overexpression was the most significant factor associated with OS after resection of HCC, and was more significant than other factors such as tumor size, vascular invasion, and tumor capsule invasion, which were also associated with OS (32). In the present study, however, we could not detect a prognostic role of $\mathrm{p} 53$ for survival in patients with NAFLD.

The retrospective design of this study and the limited sample size, especially of patients with pure NAFLD, are 
Table 4 Factors associated with survival in patients with HCC associated with NAFLD

\begin{tabular}{|c|c|c|}
\hline Characteristics & Local RFS & Systemic RFS \\
\hline Age $>65$ years & 0.501 & 0.544 \\
\hline Male sex & 0.220 & 0.119 \\
\hline Heart disease & 0.453 & 0.513 \\
\hline Hypertension & 0.515 & 0.067 \\
\hline DM & 0.410 & 0.789 \\
\hline Hyperlipidemia & 0.203 & 0.760 \\
\hline Obesity & 0.110 & 0.920 \\
\hline Child-Pugh & 0.735 & 0.715 \\
\hline MELD >10 & 0.956 & 0.600 \\
\hline AFP $>20 \mathrm{ng} / \mathrm{mL}$ & 0.986 & 0.473 \\
\hline Hypoalbuminemia & 0.796 & 0.338 \\
\hline Thrombocytopenia & 0.920 & 0.659 \\
\hline Major resection & 0.852 & 0.263 \\
\hline Anatomical resection & 0.178 & 0.459 \\
\hline Operation time $\geq 200 \mathrm{~min}$ & 0.402 & 0.322 \\
\hline Blood loss $>500 \mathrm{~mL}$ & 0.414 & 0.877 \\
\hline Blood transfusion & 0.627 & 0.355 \\
\hline Cirrhosis & 0.117 & 0.375 \\
\hline Ro & 0.428 & 0.715 \\
\hline Microvascular invasion & 0.997 & 0.415 \\
\hline Serosal invasion & 0.696 & 0.085 \\
\hline ES grade & 0.185 & 0.106 \\
\hline T stage & 0.158 & $<0.001$ \\
\hline
\end{tabular}

HCC, hepatocellular carcinoma; NAFLD, non-alcoholic fatty liver disease; RFS, recurrence-free survival; DM, diabetes mellitus; MELD, Model for End-Stage Liver Disease; AFP, $\alpha$-fetoprotein; ES, Edmonson-Steiner.

the major limitations of this study. However, even though number of patients with NAFLD associated HCC are increasing, the overall number is not very high world over and a timely evaluation of outcomes of NAFLD associated HCC was required hence we went ahead with our evaluation. To the best of our knowledge, however, this study is the first of its kind to evaluate the pathological prognostic factors in patients with NAFLD. Cirrhosis is an important limiting factor in the study leading to bias. Since evaluation of patients with NAFLD and cirrhosis reduced the number of patients

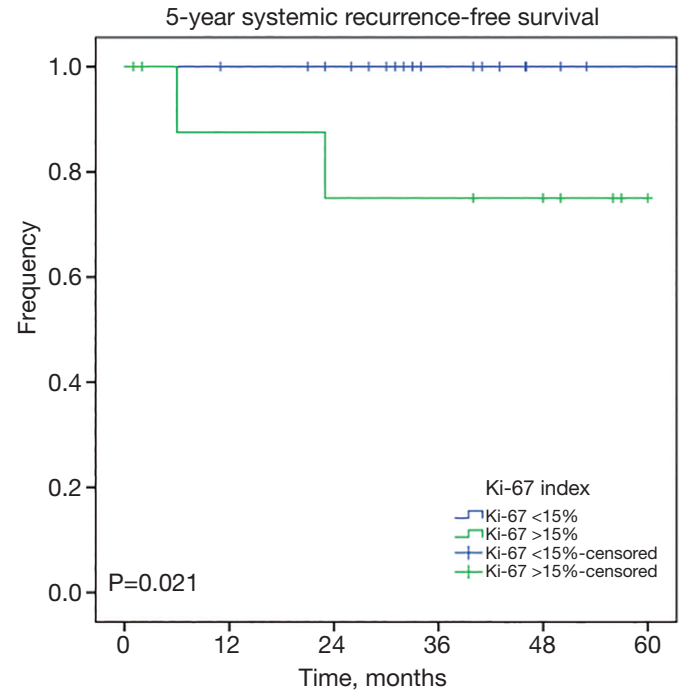

Figure 3 Systemic RFS curves for patients with NAFLD divided by the Ki-67 labeling index ( $<15 \%$ or $>15 \%)$. RFS, recurrence-free survival; NAFLD, non-alcoholic fatty liver disease.

further, we were not able to accurately analyze this subgroup. Further studies on this topic are necessary to validate our results. In particular, a large-scale multicenter study will provide greater value in this setting.

\section{Conclusions}

HCC in patients with NAFLD can occur even in the absence of cirrhosis. Molecular markers are important to predict the survival of patients with HCC. In patients with HCC associated with NAFLD, the Ki-67 labeling index determined by immunohistochemistry may help to predict the risk of post-resection extrahepatic recurrence, and allow for stricter monitoring protocols for such patients.

\section{Acknowledgments}

Funding: None.

\section{Footnote}

Reporting Checklist: The authors have completed the STROBE reporting checklist. Available at https://dx.doi. org/10.21037/tcr-21-707

Data Sharing Statement: Available at https://dx.doi. org/10.21037/tcr-21-707 
Peer Review File: Available at https://dx.doi.org/10.21037/ tcr-21-707

Conflicts of Interest: All authors have completed the ICMJE uniform disclosure form (available at https://dx.doi. org/10.21037/tcr-21-707). The authors have no conflicts of interest to declare.

Ethical Statement: The authors are accountable for all aspects of the work in ensuring that questions related to the accuracy or integrity of any part of the work are appropriately investigated and resolved. The study was conducted in accordance with the Declaration of Helsinki (as revised in 2013). The study was approved by institutional review board at Seoul National University Bundang Hospital (South Korea) (IRB No. B-2007/625/107) and individual consent for this retrospective analysis was waived.

Open Access Statement: This is an Open Access article distributed in accordance with the Creative Commons Attribution-NonCommercial-NoDerivs 4.0 International License (CC BY-NC-ND 4.0), which permits the noncommercial replication and distribution of the article with the strict proviso that no changes or edits are made and the original work is properly cited (including links to both the formal publication through the relevant DOI and the license). See: https://creativecommons.org/licenses/by-nc-nd/4.0/.

\section{References}

1. Conway B, Rene A. Obesity as a disease: no lightweight matter. Obes Rev 2004;5:145-51.

2. Perumpail RB, Wong RJ, Ahmed A, et al. Hepatocellular carcinoma in the setting of non-cirrhotic nonalcoholic fatty liver disease and the metabolic syndrome: US experience. Dig Dis Sci 2015;60:3142-8.

3. Byrne CD, Targher G. NAFLD: a multisystem disease. J Hepatol 2015;62:S47-64.

4. Hydes TJ, Ravi S, Loomba R, et al. Evidence-based clinical advice for nutrition and dietary weight loss strategies for the management of NAFLD and NASH. Clin Mol Hepatol 2020;26:383-400.

5. Sookoian S, Pirola CJ. Precision medicine in nonalcoholic fatty liver disease: new therapeutic insights from genetics and systems biology. Clin Mol Hepatol 2020;26:461-75.

6. Kleiner DE, Brunt EM, Van Natta M, et al. Design and validation of a histological scoring system for nonalcoholic fatty liver disease. Hepatology 2005;41:1313-21.
7. El Jabbour T, Lagana SM, Lee H. Update on hepatocellular carcinoma: Pathologists' review. World J Gastroenterol 2019;25:1653-65.

8. Yoo JJ, Kim W, Kim MY, et al. Recent research trends and updates on nonalcoholic fatty liver disease. Clin Mol Hepatol 2019;25:1-11.

9. Akuta N, Kawamura Y, Arase Y, et al. Hepatocellular carcinoma is the most common liver-related complication in patients with histopathologically-confirmed NAFLD in Japan. BMC Gastroenterol 2018;18:165.

10. Gentil Perret A, Mosnier JF, Buono JP, et al. The relationship between MIB-1 proliferation index and outcome in pancreatic neuroendocrine tumors. Am J Clin Pathol 1998;109:286-93.

11. Davison AC, Hinkley DV. Bootstrap Methods and their Application. Cambridge Series in Statistical and Probabilistic Mathematics. Cambridge: Cambridge University Press, 1997. Available online: https://www. cambridge.org/core/books/bootstrap-methods-and-theirapplication/ED2FD043579F27952363566DC09CBD6A

12. Anstee QM, Reeves HL, Kotsiliti E, et al. From NASH to HCC: current concepts and future challenges. Nat Rev Gastroenterol Hepatol 2019;16:411-28.

13. Liew ZH, Goh GBB, Hao Y, et al. Comparison of hepatocellular carcinoma in patients with cryptogenic versus hepatitis B etiology: a study of 1079 cases over 3 decades. Dig Dis Sci 2019;64:585-90.

14. Kollmann D, Selzner N, Selzner M. Bridging to liver transplantation in HCC patients. Langenbecks Arch Surg 2017;402:863-71.

15. Inchingolo R, Posa A, Mariappan M, et al. Locoregional treatments for hepatocellular carcinoma: current evidence and future directions. World J Gastroenterol 2019;25:4614-28.

16. Lurje I, Czigany Z, Bednarsch J, et al. Treatment strategies for hepatocellular carcinoma - a multidisciplinary approach. Int J Mol Sci 2019;20:1465.

17. Shiota G, Kishimoto Y, Suyama A, et al. Prognostic significance of serum anti-p53 antibody in patients with hepatocellular carcinoma. J Hepatol 1997;27:661-8.

18. Wellmann A, Flemming P, Behrens P, et al. High expression of the proliferation and apoptosis associated CSE1L/CAS gene in hepatitis and liver neoplasms: Correlation with tumor progression. Int J Mol Med 2001;7:489-94

19. Ito $Y$, Matsuura N, Sakon M, et al. Both cell proliferation and apoptosis significantly predict shortened diseasefree survival in hepatocellular carcinoma. Br J Cancer 
1999;81:747-51.

20. Than NN, Ghazanfar A, Hodson J, et al. Comparing clinical presentations, treatments and outcomes of hepatocellular carcinoma due to hepatitis $\mathrm{C}$ and nonalcoholic fatty liver disease. QJM 2017;110:73-81.

21. Gerdes J, Schwab U, Lemke H, et al. Production of a mouse monoclonal antibody reactive with a human nuclear antigen associated with cell proliferation. Int J Cancer 1983;31:13-20.

22. El-Bendary M, Elalfy H, Zalata K. Predictors of hepatocyte proliferative activity in chronic hepatitis $\mathrm{B}$ and $\mathrm{C}$ vs. steatohepatitis as assessed by the monoclonal antibody MIB1-Ki-67. Arab J Gastroenterol 2011;12:119-24.

23. Scholzen T, Gerdes J. The Ki-67 protein: from the known and the unknown. J Cell Physiol 2000;182:311-22.

24. Luo Y, Ren F, Liu Y, et al. Clinicopathological and prognostic significance of high Ki-67 labeling index in hepatocellular carcinoma patients: a meta-analysis. Int J Clin Exp Med 2015;8:10235-47.

25. Hussein O, Szvalb S, Van den Akker-Berman LM, et al. Liver regeneration is not altered in patients with nonalcoholic steatohepatitis (NASH) when compared to chronic hepatitis $\mathrm{C}$ infection with similar grade of inflammation. Dig Dis Sci 2002;47:1926-31.

26. Yang C, Su H, Liao X, et al. Marker of proliferation Ki-

Cite this article as: D'Silva M, Na HY, Cho JY, Han HS, Yoon YS, Lee HW, Lee JS, Lee B, Kim M. Pathological prognostic factors for post-resection survival in patients with hepatocellular carcinoma associated with non-alcoholic fatty liver disease. Transl Cancer Res 2021;10(7):3345-3355. doi: $10.21037 /$ tcr-21-707
67 expression is associated with transforming growth factor beta 1 and can predict the prognosis of patients with hepatic B virus-related hepatocellular carcinoma. Cancer Manag Res 2018;10:679-96.

27. Li HH, Qi LN, Ma L, et al. Effect of KI-67 positive cellular index on prognosis after hepatectomy in Barcelona Clinic Liver Cancer stage A and B hepatocellular carcinoma with microvascular invasion. Onco Targets Ther 2018;11:4747-54.

28. Mann CD, Neal CP, Garcea G, et al. Prognostic molecular markers in hepatocellular carcinoma: A systematic review. Eur J Cancer 2007;43:979-92.

29. Qin LX, Tang ZY. The prognostic molecular markers in hepatocellular carcinoma. World J Gastroenterol 2002;8:385-92.

30. Jeng KS, Sheen IS, Chen BF, et al. Is the p53 gene mutation of prognostic value in hepatocellular carcinoma after resection? Arch Surg 2000;135:1329-33.

31. Heinze T, Jonas S, Kärsten A, et al. Determination of the oncogenes p53 and C-erb B2 in the tumour cytosols of advanced hepatocellular carcinoma (HCC) and correlation to survival time. Anticancer Res 1999;19:2501-3.

32. Qin LX, Tang ZY, Ma ZC, et al. P53 immunohistochemical scoring: an independent prognostic marker for patients after hepatocellular carcinoma resection. World J Gastroenterol 2002;8:459-63. 